\title{
Análisis de las variables meteorológicas registradas por la estación automática de la Universidad Continental en Huancayo, 2015
}

\author{
Analysis of meteorological variables recorded by the Universidad \\ Continental automatic station in Huancayo, 2015
}

\author{
René Estevan Arredondo \\ Universidad Continental \\ realcmw@gmail.com
}

\section{RESUMEN}

Este trabajo tiene como objetivo analizar el comportamiento de las variables meteorológicas registradas por la estación automática instalada en la Universidad Continental, durante el período de abril y septiembre de 2015. En ésta investigación se determinaron los principales estadígrafos de las variables estudiadas, así como las tendencias de las mismas. A partir de los estadígrafos, tendencias y las figuras elaboradas para cada variable, se analizaron las principales características que describen el comportamiento de cada una de las variables registradas, dentro del período estudiado. Durante este período han existido irregularidades con algunos sensores, fundamentalmente el de radiación solar, lo cual limita en cierta medida los resultados aquí obtenidos. El potencial solar medio calculado para este estudio alcanza la magnitud de 5,1 kWh m-2 día ${ }^{-1}$, siendo agosto el mes de mayor potencial. El viento predominante es de rumbo norte (N). La velocidad media del viento es de 1,07 $\mathrm{m} \mathrm{s}^{-1}$ y la velocidad máxima registrada tuvo lugar el 6 de mayo con una magnitud de $10,3 \mathrm{~m} \mathrm{~s}^{-1}$ con rumbo norte (N). Puede considerarse, teniendo en cuenta el límite temporal de esta investigación, que el 2015 ha sido un año más cálido, cuando se compara con los valores medios mensuales calculados para mayores períodos de tiempo. El régimen de precipitaciones muestra una distribución anómala en comparación con otras investigaciones que abordan mayores períodos de tiempos, no obstante, los valores acumulados se encuentran en el mismo orden de magnitud.

Palabras clave: Temperatura, presión, irradiancia, potencial solar, tendencias.

\begin{abstract}
The objective is to analyze the meteorological variables behavior recorded by the installed automatic station in the Universidad Continental, during the April and September 2015 period. The research determine the main statisticians of the studied variables, as well as its trends. From statisticians, trends and made figures for each variable, the main characteristics which describe each registered variables behavior during the analyzed period were analyzed. During this period there have been irregularities with some sensors, mainly with the solar radiation, limiting in some way the obtained results. The average solar potential calculated for this research reaches the magnitude of $5,1 \mathrm{kWh} \mathrm{m}^{-2}$ day $^{-1}$, being august the month with the greatest potential value. The prevailing wind is in the north direction $(\mathrm{N})$. The average wind speed is only $1,07 \mathrm{~m} \mathrm{~s}^{-1}$ and the maximum recorded speed took place on May 6 with a magnitude of $10,3 \mathrm{~m} \mathrm{~s}^{-1}$ to the north $(\mathrm{N})$ direction. It may be considered, taking into account the time limit of this research, that 2015 was a warmer year, when compared with the monthly average values calculated for longer periods of time. The precipitations regime shows an anomalous distribution compared to other researchs addressing longer periods of time, however, the accumulated values are in the same magnitude order.
\end{abstract}

Keywords: Temperature, pressure, irradiance, solar potential, trends.

Historial del artículo:

Recibido: 14 de agosto de 2015. Aprobado: 8 de octubre de 2015. Disponible en línea: 30 de diciembre de 2015

1 Doctor en Ciencias Meteorológicas, Investigador de la Universidad Continental. 


\section{INTRODUCCIÓN}

La Estación Meteorológica Automática (EMA) de la Universidad Continental se encuentra ubicada en los $12^{\circ} 02^{\prime} 75^{\prime \prime}$ de latitud Sur y los $75^{\circ} 11^{\prime} 51^{\prime \prime}$ de longitud Oeste, a una altitud de 3307 m s.n.m. en la ciudad de Huancayo - Perú. Los datos generados por esta estación son empleados en el marco del proyecto: "Estudio de la variación de las propiedades ópticas de los aerosoles debido a las quemas de vegetación para evaluar su influencia en el forzamiento radiativo terrestre"; el cual tiene entre sus objetivos evaluar el efecto que provocan los aerosoles atmosféricos generados por la quema de biomasa en los procesos de transferencia radiativa de la atmósfera $(1,2,3,4)$.

Las mediciones que se realizaron con la EMA juegan un importante papel dentro de la investigación, fundamentalmente las mediciones de radiación solar en superficie.

Al no existir antecedentes de trabajos similares para la

\section{MATERIAL Y MÉTODOS}

Esta investigación es de nivel descriptivo comparativo y para ella se ha contado con la EMA marca Hobo Energy que en el mes de febrero de 2015 fue instalada en el campus de la Universidad Continental. Esta estación fue situada a una altura aproximada de 27,5 $\mathrm{m}$ sobre el nivel del suelo. En la tabla 1 aparecen los sensores que forman parte de dicha estación, así como, las principales características técnicas de los mismos. Estos sensores cuentan con la correspondiente calibración realizada en fábrica. Las variables de la estación son registradas cada 5 min durante las 24 h. En total, se registran 288 valores de cada variable. Hasta el momento, las mediciones son almacenadas en el "datalogger" y semanalmente descargadas y analizadas.

Los datos registrados entre febrero y marzo de 2015, no son empleados en el presente estudio debido a la inestabilidad de las mediciones como consecuencia de las reiteradas interrupciones por problemas técnicos

Tabla № 1: Tipos y características de los sensores de la Estación Meteorológica Automática de la Universidad Continental.

\begin{tabular}{llccc}
\hline \multicolumn{1}{c}{ Sensor } & \multicolumn{1}{c}{ Modelo } & Rango & Exactitud & Resolución \\
\hline Barómetro & S-BPA-CM10 & $600-1070 \mathrm{mbar}$ & $\pm 3 \mathrm{mbar}$ & $0,1 \mathrm{mbar}$ \\
Temperatura & S-THB-MOOX & $-40-75^{\circ} \mathrm{C}$ & $\pm 0,21^{\circ} \mathrm{C}$ & $0,02{ }^{\circ} \mathrm{C}$ \\
Humedad Relativa & S-THB-MOOX & $0-100 \%$ & $\pm 2,5 \%$ & $0,1 \%$ \\
Pluviómetro & S-RGA-MO03 & $0-12,7 \mathrm{~cm} \mathrm{~h}^{-1}$ & $\pm 1 \%$ & $0,2 \mathrm{~mm}$ \\
Piranómetro & S-LIB-M003 & $0-1280 \mathrm{~W} \mathrm{~m}^{-2}$ & $\pm 10 \mathrm{~W} \mathrm{~m}^{-2}$ & $1,24 \mathrm{~W} \mathrm{~m}^{-2}$ \\
Viento (velocidad) & S-WSET-B & $0-76 \mathrm{~m} \mathrm{~s}^{-1}$ & $\pm 4 \%$ & $0,5 \mathrm{~m} \mathrm{~s}^{-1}$ \\
Viento (dirección) & S-WSET-B & $0-355^{\circ}$ & $\pm 5^{\circ}$ & $1,4^{\circ}$ \\
\hline
\end{tabular}

ciudad de Huancayo, los resultados obtenidos en este estudio son novedosos.

Para el análisis del comportamiento de cada una de las variables, se determinaron sus respectivos valores medios, máximos y mínimos, así como, sus desviaciones estándar. Se graficaron las series de datos para el período comprendido entre los meses de abril y septiembre de 2015 para cada variable y se establecieron sus respectivas tendencias.

En la bibliografía consultada se han encontrado caracterizaciones de algunas variables meteorológicas, fundamentalmente temperatura y precipitación, en la cuenca del río Mantaro, para diversos períodos de tiempo $(5,6)$. Se han realizado también estudios de las temperaturas extremas en el Valle del Mantaro (7), así como, análisis de tendencias climáticas de las temperaturas y las precipitaciones (8), empleando en ambos casos las mediciones realizadas en el Observatorio de Huancayo, ubicada a $12^{\circ} 02^{\prime} 18^{\prime \prime} S$, $75^{\circ} 19^{\prime} 22^{\prime \prime} \mathrm{W}, 3350$ m s.n.m. de algunos de sus sensores. Por tanto, en el presente trabajo se emplearon los datos registrados desde el 1ro de abril de 2015 hasta el 30 de septiembre del mismo año. No obstante, existen irregularidades en las series de datos debido a los problemas técnicos de algunos sensores en determinados períodos de tiempo.

Excepto para las mediciones de la Presión Atmosférica y la Fuerza y Dirección del Viento, en el resto de las variables registradas existen faltantes de datos. En el caso de la Temperatura y la Humedad Relativa, los datos faltantes corresponden a los días comprendidos entre el 3 y 10 del mes de julio para un 95,6\% de completamiento. En el caso de la Precipitación faltan un total de 15 días de datos entre el 22 de abril y el 6 de mayo para un 91,8 \% de completamiento. La variable con menos datos es la de Radiación Solar con un 68,85\%, en total, 57 días faltantes de 183 posibles. Excepto en el mes de agosto, con el $100 \%$ de los registros, en todos los meses restantes existen días faltantes, en mayor o menor cuantía. El mes de junio sigue al mes de agosto en cuanto a completamiento 
Tabla $N^{\circ}$ 2: $\quad$ Valores medios de los principales estadígrafos de las variables meteorológicas, calculadas y sus respectivas Unidades de Medida (UM).

\begin{tabular}{|c|c|c|c|c|c|}
\hline Variable & Media & Mínimo & Máximo & $\begin{array}{c}\text { Desv. } \\
\text { Estándar }\end{array}$ & UM \\
\hline Presión & 686,52 & 683,62 & 688,75 & 688,75 & $\mathrm{hPa}$ \\
\hline Temperatura & 12,28 & 6,29 & 20,57 & 20,57 & ${ }^{\circ} \mathrm{C}$ \\
\hline Humedad Relativa & 60,42 & 34,07 & 81,40 & 81,40 & $\%$ \\
\hline Viento (fuerza) & 1,07 & - & 5,74 & 5,74 & $\mathrm{~m} / \mathrm{s}$ \\
\hline Irradiancia & 449,94 & - & 1009,66 & 1009,66 & $\mathrm{~W} / \mathrm{m}^{2}$ \\
\hline
\end{tabular}

ya que solamente no se registraron datos durante los cuatro primeros días de ese mes.

Se determinaron los comportamientos medios mensuales de cada una de las variables, así como, el comportamiento medio diurno de las mismas, por meses y para todo el período. coincidiendo con la época de invierno en la región (5, $6,7,8)$.

De lo expresado puede afirmarse que, durante el invierno, sobre la región, predominan presiones más altas que durante el verano. No obstante, el valor máximo absoluto de la presión se registró el 18 de

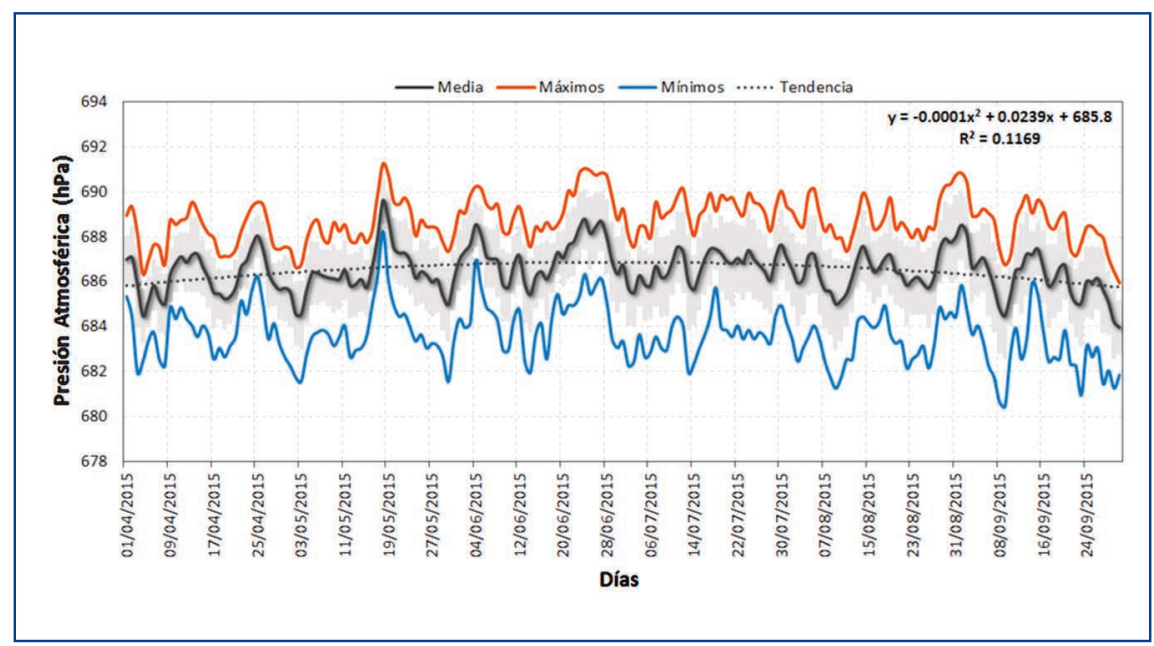

Figura $\mathrm{N}^{\circ}$ 1: Comportamiento de la presión media diaria y de las mínimas y máximas del día durante el período analizado. La desviación estándar se muestra en la franja de color gris.

\section{RESULTADOS}

En la tabla 2, se muestran a modo de resumen, los principales estadígrafos calculados a partir de los valores medios diarios de las variables analizadas.

El comportamiento de los valores medios diarios de la Presión Atmosférica, así como, de los valores máximos y mínimos diarios, y de las correspondientes desviaciones estándar para el período objeto de estudio, se muestran en la figura 1. Igualmente se representa el ajuste de segundo orden mediante el cual se describe el comportamiento de la presión a lo largo del período analizado. En los extremos de este ajuste se encuentran los mínimos mientras que hacia el centro del gráfico se encuentran los valores más altos del ajuste. Este máximo del ajuste tiene lugar a finales del mes de junio y primeros días del mes de julio mayo entre las 9:25 y las 9:30 Hora Local (HL). En esta fecha tiene lugar también la menor amplitud en la variación diurna de la presión, con apenas $3 \mathrm{hPa}$ de diferencia entre la mínima y máxima presión para un día dentro de todo el período analizado, claramente apreciable en el gráfico. La mayor amplitud en los valores de presión, con una diferencia de $7 \mathrm{hPa}$, fue registrada los días 13 de julio y 7 de septiembre, próxima a esta última fecha, exactamente el día 9 de septiembre, entre las 16:00 y las 16:10 HL tiene lugar el mínimo valor de presión para todo el período.

En la figura 2 se representan los valores medios diarios de la temperatura del aire, así como, los valores mínimos y máximos para cada día del período analizado, se muestra además la desviación estándar correspondiente a cada día. Como fue expresado anteriormente, entre el 3 y 10 de julio, debido a 
problemas técnicos del sensor de temperatura, esta variable se dejó de registrar. La tendencia de las temperaturas medias diarias, representadas con la línea de puntos, muestra una muy ligera pendiente ascendente, a razón de $0,0063{ }^{\circ} \mathrm{C}$ por día. El mínimo valor de la temperatura media $\left(8,96^{\circ} \mathrm{C}\right)$ se registró el día 5 de abril, como consecuencia de la menor amplitud dentro de la muestra, o sea, una diferencia mes de junio con una magnitud de $1,62{ }^{\circ} \mathrm{C}$ el día 8 a las 06:40 HL.

Tanto el máximo absoluto de la temperatura como el máximo de los valores medios tuvo lugar el día 27 de septiembre, el primero con un valor de $24,85{ }^{\circ} \mathrm{C}$ a las 15:55 HL, mientras que en el caso del máximo de la medias alcanzó una magnitud de $14,57^{\circ} \mathrm{C}$. Por

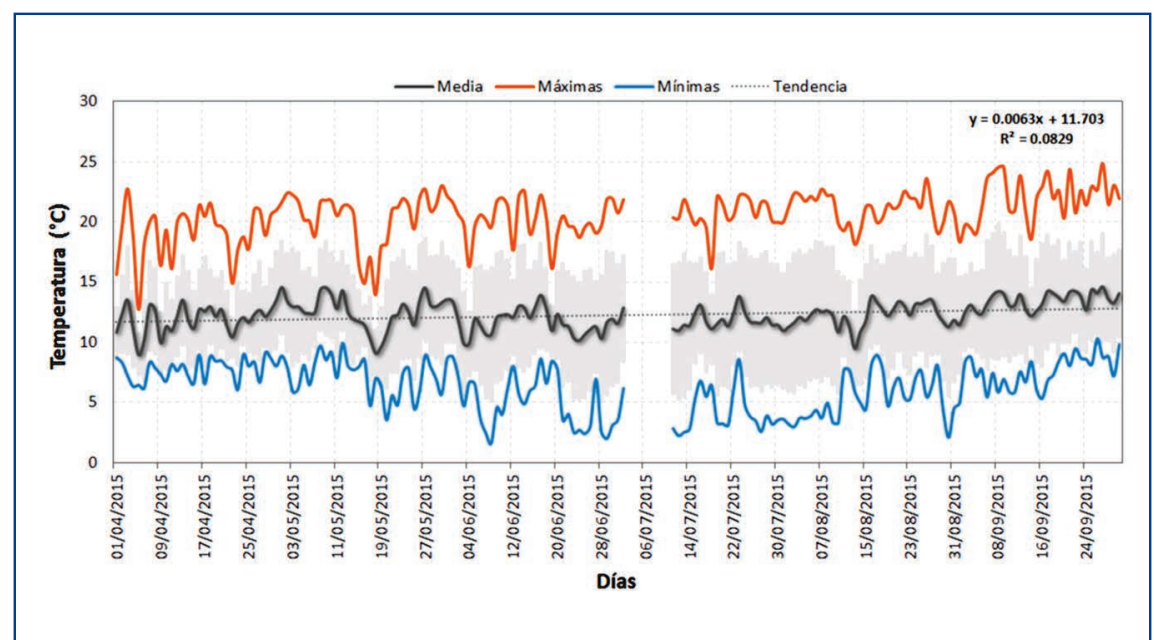

Figura $\mathrm{N}^{\circ} 2$ : Comportamiento de la temperatura media diaria y de las mínimas y máximas del día durante el período analizado. La desviación estándar se muestra en la franja de color gris.

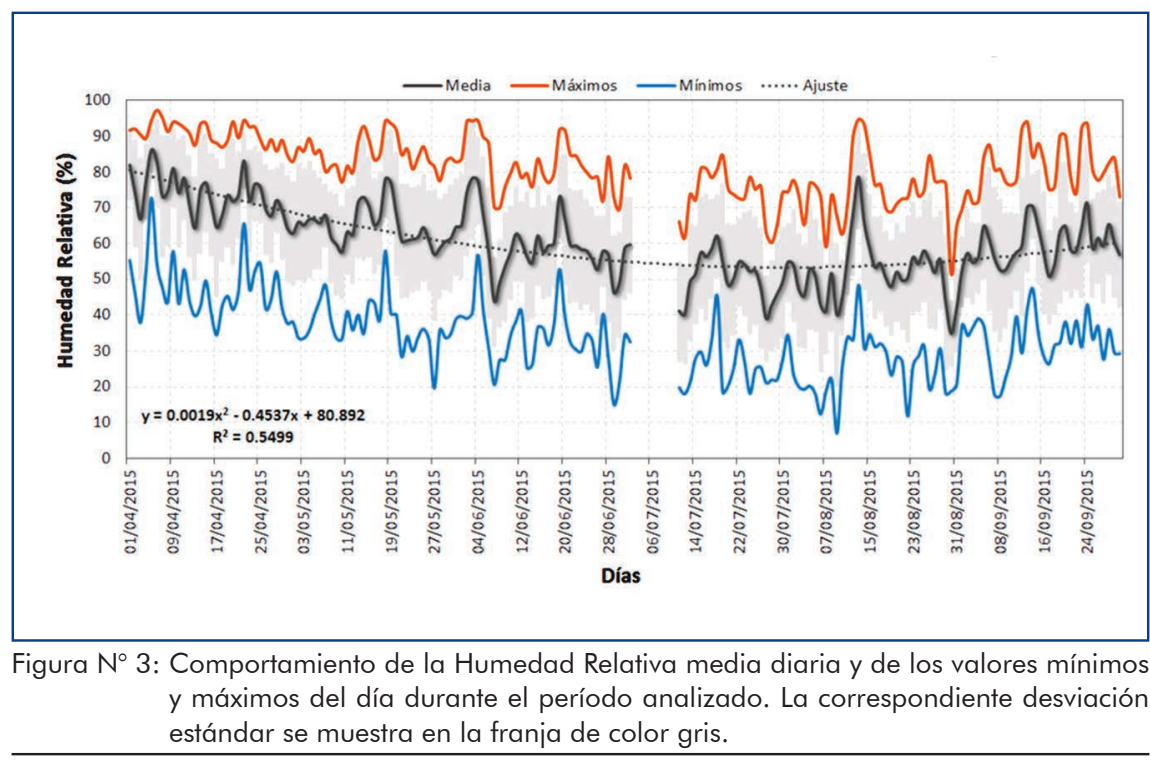

entre la mínima y la máxima temperatura para ese día de apenas $6,27^{\circ} \mathrm{C}$, cuando la amplitud promedio en el período es de $14,28^{\circ} \mathrm{C}$. Este día constituyó uno de los días más fríos del período analizado. No obstante, coincidiendo con el comportamiento de la presión y en contrafase con esta, entre los meses de junio y julio se encuentran los valores mínimos más bajos del período, con el mínimo absoluto precisamente a principios del otro lado la máxima amplitud de la temperatura ocurrió el día 29 de junio llegando a los 19,9 ${ }^{\circ} \mathrm{C}$ de diferencia, coincidiendo también con el máximo valor de la desviación estándar $(5,95)$ en el período analizado. En el caso de las temperaturas máximas es claramente apreciable una tendencia al aumento a razón de $0,016{ }^{\circ} \mathrm{C}$ por día. Sin embargo, en el caso de las mínimas, 
el comportamiento de estas se ajusta mejor a una curva polinómica de segundo orden, con los máximos en los extremos de la serie y los mínimos hacia el centro de esta, o sea, en los meses de junio y julio, precisamente en contrafase con la presión atmosférica como fue mencionado anteriormente. El mes más frío dentro del período objeto de estudio fue junio con una temperatura promedio de $11,63^{\circ} \mathrm{C}$, seguido del mes de julio con un valor medio $11,82{ }^{\circ} \mathrm{C}$. Por su parte el mes más cálido corresponde a septiembre con una temperatura promedio de $13,39^{\circ} \mathrm{C}$.

Al igual que en el caso de la temperatura, la variable Humedad Relativa (figura 3), dejó de registrarse entre los días 3 al 10 de julio, por las razones antes expuestas. La curva de ajuste de los valores medios diarios de la humedad (línea gris de puntos), muestra claramente cuál ha sido el comportamiento de esta variable durante el período analizado. Partiendo de desviación estándar, ocurren el día 19 de julio con valores de $66,2 \%$ y 21,55 , respectivamente. El valor mínimo medio de la humedad tiene lugar el 30 de agosto con una magnitud de 34,76\%. El mínimo absoluto por su parte tuvo lugar el día 9 de agosto alcanzando solamente un $7 \%$ de humedad relativa en el horario de las 17:05 HL. El mes con mayor humedad corresponde a abril con una media de $73,61 \%$, seguido del mes de mayo con $64,69 \%$. En el resto de los meses la Humedad Relativa se mantuvo por debajo del $60 \%$, con el mínimo en el mes de julio y un valor de $50,2 \%$ en este caso.

La velocidad media del viento para el período objeto de análisis, representada en la figura 4 por la curva gris, no llega a alcanzar en ninguno de los días analizados, los $2 \mathrm{~m} \mathrm{~s}^{-1}$, de hecho, el valor medio de esta variable es de apenas $1,07 \mathrm{~m} \mathrm{~s}^{-1}$ para toda la muestra. No obstante existe una muy ligera tendencia al ascenso a

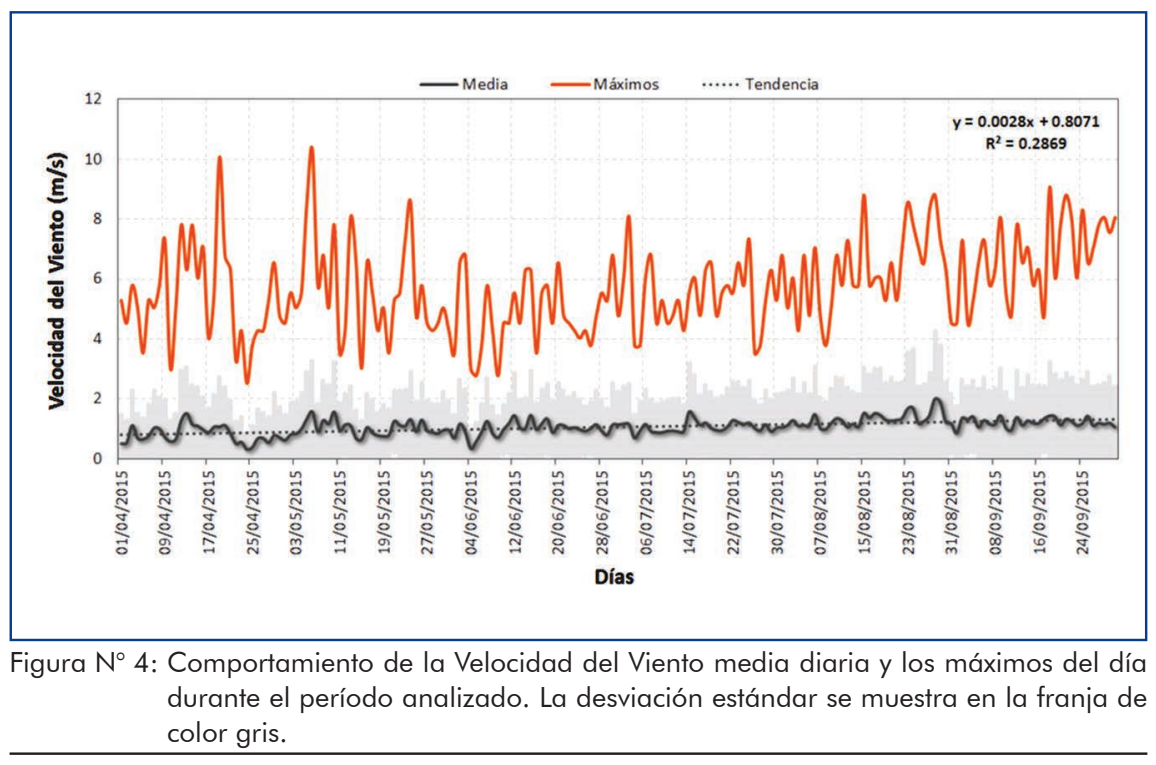

valores altos de humedad media diaria en el mes de abril, entorno al $80 \%$, disminuyendo hasta el mes de julio donde tienen lugar los valores medios mínimos, para luego comenzar nuevamente un ascenso más discreto a partir del mes de agosto y hasta finales de septiembre. Es precisamente en el mes de abril cuando tienen lugar los máximos valores de humedad. En el caso de los valores medios, el máximo tuvo lugar el día 5 con una magnitud de $86,2 \%$, coincidiendo en este caso con el mínimo valor de la desviación estándar para el período $(5,45)$ y con la menor amplitud dentro de la serie $(21,70 \%)$. Por su parte el máximo absoluto llegó a alcanzar 97,3\% el día 6 del propio mes entre las $07: 30$ y las $07: 35 \mathrm{HL}$.

Resaltan en el gráfico anterior otros dos máximos ocurridos los días 4 de junio y 13 de agosto con valores de 94,4 y $94,8 \%$, respectivamente. La mayor diferencia entre los mínimos y máximos, y la mayor razón de 0,0028 $\mathrm{m} \mathrm{s}^{-1}$ día $^{-1}$. El máximo valor medio se registró el día 28 de agosto con una magnitud de $1,99 \mathrm{~m} \mathrm{~s}^{-1}$, mientras que el máximo absoluto, con un valor de 10,32 $\mathrm{m} \mathrm{s}^{-1}$, tuvo lugar el día 6 de mayo a las 15:05 HL desde los $21,1^{\circ}$ de dirección (NNE). Excepto en el mes de abril, cuyo valor medio es de apenas 0,81 $\mathrm{m} \mathrm{s}^{-1}$, en el resto de los meses los valores medios de la velocidad del viento superan $1 \mathrm{~m} \mathrm{~s}^{-1}$, siendo el mes de mayor velocidad media del viento agosto con un valor de $1,31 \mathrm{~m} \mathrm{~s}^{-1}$. Los valores máximos diarios muestran también una tendencia ascendente, pero en este, caso con una pendiente ligeramente superior $\left(0,009 \mathrm{~m} \mathrm{~s}^{-1}\right)$ respecto a la del viento medio diario.

Las direcciones del viento predominantes para el período objeto de estudio, se representan en la figura 5 , a través de las rosas de los vientos, tanto en 8 como en 16 rumbos. En ambos casos el viento predominante es del norte $(\mathrm{N})$ con un $46,34 \%$ de frecuencia de 
ocurrencia para 8 rumbos y de $33,51 \%$ para 16 rumbos. En el caso de 8 rumbos la velocidad media del viento de la dirección norte es de 1,07 $\mathrm{m} \mathrm{s}^{-1}$, mientras que la velocidad máxima absoluta ocurrida para esta dirección es de 10,32 $\mathrm{m} \mathrm{s}^{-1}$. Le siguen en frecuencia de ocurrencias, la dirección noroeste (NO) con 13,19\%, un viento medio de $1,49 \mathrm{~m} \mathrm{~s}^{-1}$ y un máximo absoluto para este rumbo de 10,07 $\mathrm{m} \mathrm{s}^{-1}$, y la dirección oeste (O) con 11,97\%, el valor del viento medio es de 0,94
(N) con una velocidad media del viento de 1,09 $\mathrm{m} \mathrm{s}^{-1}$ y un viento máximo absoluto de $8,81 \mathrm{~m} \mathrm{~s}^{-1}$. Le sigue con el 10,63\% de los casos la dirección norte-noreste (NNE), con un valor de viento medio de 1,21 $\mathrm{m} \mathrm{s}^{-1}$ y en el caso del viento máximo absoluto, con una magnitud de 10,32 $\mathrm{m} \mathrm{s}^{-1}$, el cual constituye la mayor magnitud del viento máximo para todos los rumbos. El rumbo norte-noroeste (NNO) ocupa el tercer lugar en el número de casos con una frecuencia de

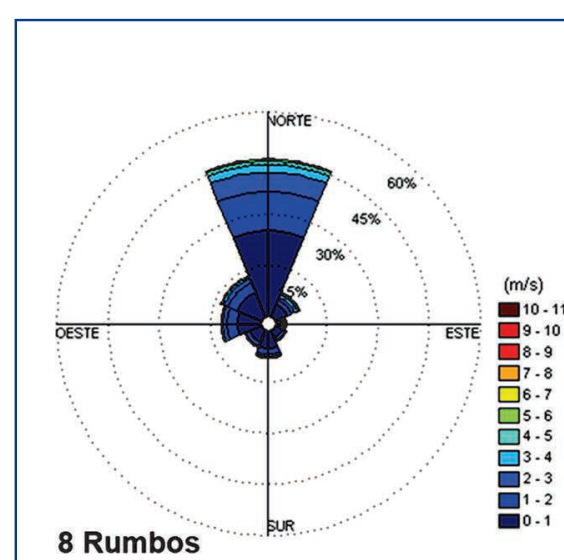

8 Rumbos

\section{Rumbos}

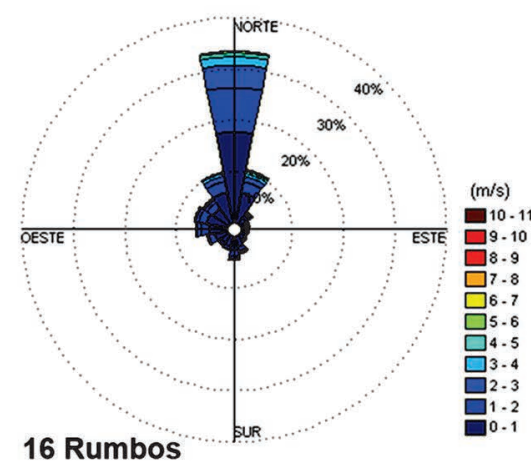

Figura $N^{\circ}$ 5: Representación de la dirección del viento predominante en 8 y 16 rumbos, as como, la distribución de las velocidades del viento, expresadas en metros por segundo, para los diferentes rumbos.

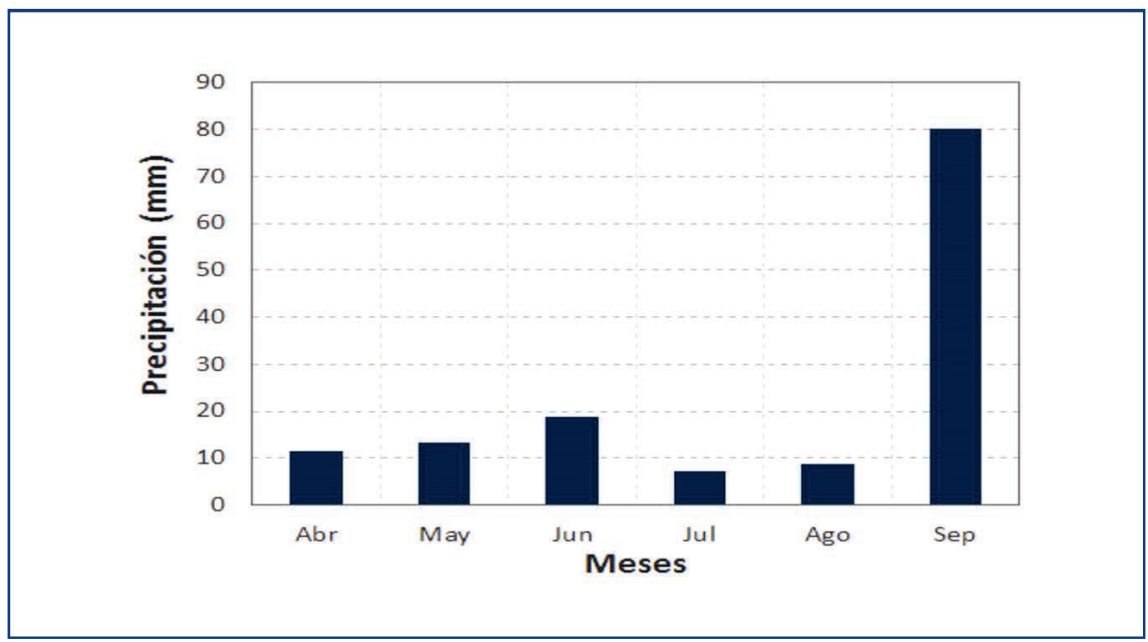

Figura $\mathrm{N}^{\circ}$ 6: Comportamiento de los acumulados mensuales de precipitación durante el período analizado. $\mathrm{m} \mathrm{s}^{-1}$ y un viento máximo absoluto de $8,56 \mathrm{~m} \mathrm{~s}^{-1}$. El resto de los rumbos no supera el $10 \%$ de ocurrencia, siendo el rumbo con menor frecuencia de ocurrencia el este $(E)$, con apenas $3,18 \%$, sin embargo, es en este rumbo donde se alcanzó el máximo valor medio de la fuerza del viento con $1,66 \mathrm{~m} \mathrm{~s}^{-1}$.

Para el caso de 16 rumbos, como fue mencionado anteriormente, el viento predominante es del norte ocurrencia de 10,12\%, un viento medio de 0,98 m $\mathrm{s}^{-1}$ y un viento máximo absoluto para este rumbo de $8,56 \mathrm{~m} \mathrm{~s}^{-1}$. El resto de los rumbos no sobrepasó el 7 $\%$ de ocurrencias. El máximo valor del viento medio tuvo lugar en el rumbo este-noreste (ENE) con una magnitud de 2,01 $\mathrm{m} \mathrm{s}^{-1}$. En la figura 6 se representan los acumulados mensuales de la precipitación, las cuales puede considerarse han sido escasas durante el período analizado. El acumulado del período es de 
$139,50 \mathrm{~mm}$, siendo el mes de septiembre el de mayor aporte, con un total de $80,21 \mathrm{~mm}$, lo que constituye el $57,5 \%$ de toda la precipitación caída en el período. Dentro de este mes la mayor contribución tuvo lugar el día 12 con un acumulado de $34,98 \mathrm{~mm}$, o sea, el $43,6 \%$ del acumulado del mes en un lapso de 4 horas con 35 minutos. El acumulado de lluvia de este día supera los acumulados mensuales registrados para cada mes entre abril y agosto. El segundo día con mayores precipitaciones se encuentra también dentro del mes de septiembre, concretamente el día 23 , con un acumulado de $13,66 \mathrm{~mm}$. Si se sumaran los acumulados de estos dos días (48,64 mm), ambos días constituirían el $34,87 \%$ de la lluvia registrada en los 183 días que incluye el período analizado, o sea, más de la cuarta parte de toda la lluvia registrada durante los 6 meses analizados.

Es importante señalar que entre los días 22 de abril y 6 hasta 1 276,90 $\mathrm{W} \mathrm{m}^{-2}$. En este caso se encuentran un total de 14 días, por lo que igualmente el análisis de los valores extremos de irradiancia se verá seriamente afectado e igualmente deberán ser tomados con cautela.

La tendencia durante el período analizado, representada en la figura por la línea gris punteada, muestra un ascenso gradual de la radiación solar a razón de $0,548 \mathrm{~W} \mathrm{~m}^{-2}$ día $^{-1}$. La media para todo el período tiene un valor de 452,24 $\pm 300,76 \mathrm{~W} \mathrm{~m}^{-2}$. Resalta en la figura el comportamiento tanto de los valores medios, como de los máximos diarios, entre el 11 de julio y el 10 de agosto donde la irradiancia muestra un comportamiento bastante lineal. Este comportamiento se debe a la existencia de muy poca nubosidad durante ese período y solamente los días 17 y 18 de julio rompen con esa linealidad tanto en los valores medios diarios como en los máximos

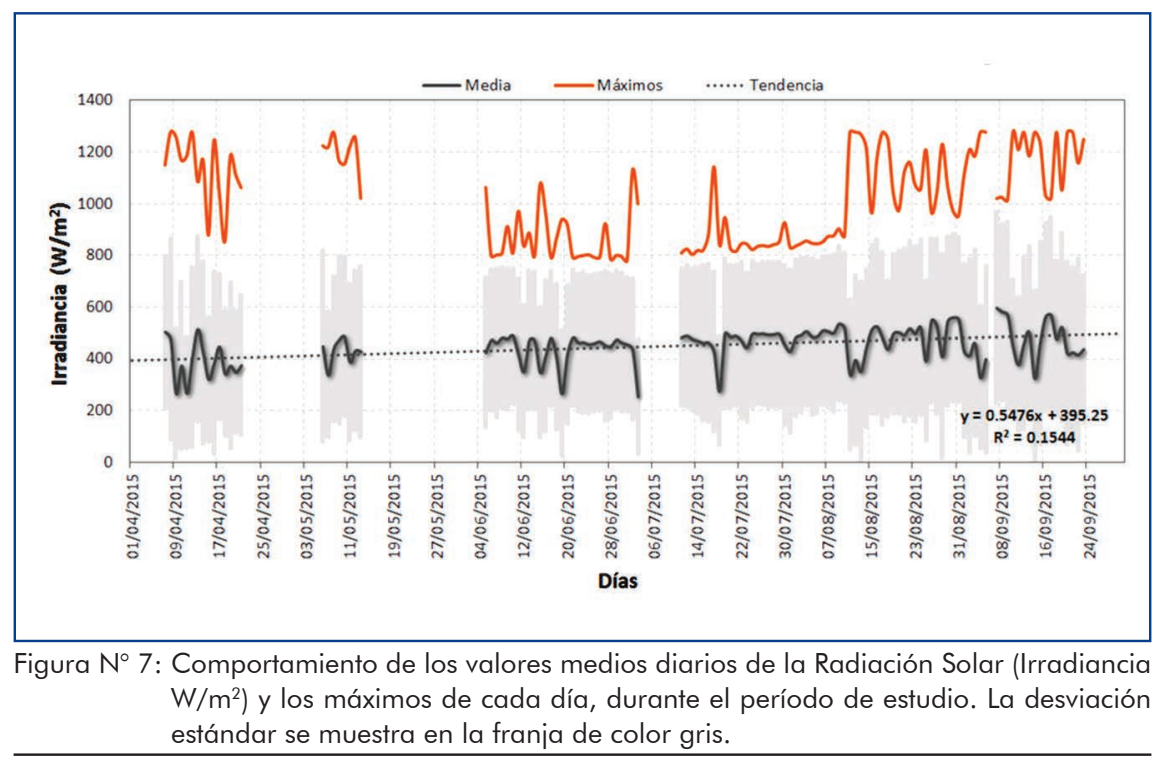

de mayo (15 días), no existen registros de precipitación debido a problemas técnicos presentados por el sensor. Independientemente de esto, en un total de 128 días (excluyendo los 15 antes mencionados), no se registraron lluvias, constituyendo el 76,19\% del período analizado. En el intervalo abril - agosto, junio es el mes con mayores precipitaciones, con un total de 18,67 mm, seguido de mayo con 13,15 mm. Por su parte, el mes con menores precipitaciones es julio con apenas $7,07 \mathrm{~mm}$, seguido de agosto con tan solo $8,8 \mathrm{~mm}$.

La variable con un bajo nivel de completamiento es la de Radiación Solar, con apenas un 68,85 \% (figura 7). Otro aspecto importante que afecta el análisis de esta variable está relacionado con los valores máximos. Debido a que el sensor utilizado cubre solamente el rango comprendido entre los 0 y los $1280 \mathrm{~W} \mathrm{~m}^{-2}$, los máximos registrados por la estación llegan solamente diarios de la irradiancia. En el caso del día 17 ocurre un incremento en el valor de los máximos debido a la presencia de nubosidad, no opaca, de forma intermitente a partir del mediodía. Por el contrario el día 18 del mismo mes, a partir de las 10:50 HL, ocurre una abrupta caída de la irradiancia, más de $640 \mathrm{~W} \mathrm{~m}^{-2}$, debido a la presencia de nubes opacas de forma permanente durante el resto del día, no dejando intersticios para que la irradiancia directa y la difusa atmosférica alcancen la superficie. Como consecuencia, se registra un mínimo en los valores medios diarios de irradiancia con una magnitud de $277,15 \mathrm{~W} \mathrm{~m}^{-2}$. A partir de esta última fecha comienza a incrementarse la presencia de nubosidad lo que provoca incrementos de los valores máximos diarios debido a la contribución de la radiación difusa consecuencia de la dispersión provocada por las nubes $(9,10)$. 


\section{DISCUSIÓN}

Las temperaturas mínimas medias mensuales presentan en este estudio un comportamiento similar al descrito por diversos autores para el valle del Mantaro, en general $(5,6)$ y para el Observatorio de Huancayo en particular $(7,8)$. Estos estudios han abarcado diversos períodos de tiempo, coincidiendo todos en un descenso desde los primeros meses del año hasta el mes de julio, donde se alcanzan los valores mínimos, para luego iniciar un ascenso hasta el último mes analizado en este estudio (septiembre). Sin embargo, en cuanto a las magnitudes, las medias mensuales de la temperatura mínima en Huancayo para el período analizado, son superiores como promedio, en el orden de los $3{ }^{\circ} \mathrm{C}$, a las medias mensuales de los períodos $1971-2000$ y 2001 - 2010 (5). En el mismo orden de magnitud se encuentran las diferencias con respecto a los períodos 1992 - 2009 (7) y 1921 - 2010 (8).

En el caso de las medias mensuales de la temperaturas máximas, no ocurre igual que con las mínimas, ya que el comportamiento no es similar a los estudios antes citados. En todos estos estudios $(5,7,8)$ las máximas medias alcanzan un máximo secundario en el mes de mayo, para luego decrecer hasta el mes de julio donde ocurre un mínimo de estas máximas, luego se incrementan nuevamente hasta alcanzar el máximo principal dentro del año en el mes de noviembre. En el presente estudio el valor más bajo de las máximas medias ocurre en el mes de junio y en el mes julio, el valor máximo medio es incluso superior al del mes de mayo en 31 décimas de grados. Las diferencias medias llegan a ser de $2,5{ }^{\circ} \mathrm{C}$ respecto a (5) y en el orden un grado comparado con (8), la menor diferencia se encontró respecto a (7) con una magnitud solamente de $0,4{ }^{\circ} \mathrm{C}$.

El régimen de precipitaciones determinado en el presente estudio difiere de los obtenidos para la década 2001 - 2010 (5) y para el período 1921 2010 (8). En el caso de estos dos períodos antes mencionados, el régimen de precipitaciones entre los meses de abril y septiembre, rango de meses empleados en este trabajo, son exactamente iguales, con pequeñas diferencias solamente en las magnitudes. El comportamiento típico de las precipitaciones de acuerdo a las medias de los acumulados para los períodos antes mencionados muestra un máximo principal en el mes de abril, descendiendo hasta el mes de julio donde ocurre el mínimo para luego comenzar a ascender hasta el mes de septiembre donde tiene lugar un máximo secundario. Por su parte los resultados derivados del presente estudio muestran que al mes de septiembre como máximo principal y a junio como máximo secundario. El mes de abril es el tercer mes de este período con menores precipitaciones, precedido por agosto y julio, en este último mes tiene lugar el mínimo acumulado de la serie. A pesar de que los regímenes de precipitaciones difieren, los acumulados son muy similares, resultando ligeramente inferior en aproximadamente $10 \mathrm{~mm}$ (8) y $20 \mathrm{~mm}$ (5), respectivamente.

Teniendo en cuenta las limitaciones de la información sobre radiación solar, manifestadas en epígrafes anteriores se ha determinado que los valores medios mensuales del potencial solar registrados manifiestan una tendencia ascendente a razón de 0,25 $\mathrm{kWh} \mathrm{m}^{-2}$ día ${ }^{-1}$, llegando a su máximo valor en el mes de agosto con una magnitud de $5,75 \mathrm{kWh} \mathrm{m}^{-2}$ día $^{-1}$. El valor medio para el período analizado es de $5,1 \mathrm{kWh} \mathrm{m}^{-2}$ día $^{-1}$, inferior a los reportados en otros trabajos (11, 12). No obstante, de acuerdo a estos estudios los máximos valores de irradiancia se registran alrededor del mes de noviembre de 2015.

A modo de conclusiones cabe señalar que en general, puede calificarse el período analizado como más cálido respecto a los valores medios reportados en otros trabajos. Igualmente, las precipitaciones muestran un comportamiento atípico en relación con valores medios históricos para diferentes períodos de tiempo, aunque los acumulados son similares en el mismo período de tiempo. El potencial de la radiación solar puede considerarse alto en concordancia con otros análisis para la región donde se encuentra ubicada la EMA.

\section{Agradecimientos}

Al CONCYTEC por el apoyo financiero para esta investigación a través del proyecto "Estudio de la variación de las propiedades ópticas de los aerosoles debido a las quemas de vegetación para evaluar su influencia en el forzamiento radiativo terrestre", contrato $N^{\circ}$ 010-2013-FONDECYT.

\section{REFERENCIAS BIBLIOGRÁFICAS}

1. Penner JE, Dickinson RE, O'Neill CA. Effects of Aerosol from Biomass Burning on the Global Radiation Budget. Science. 1992; 256: 14321433.

2. Chylek P, Wong P. Effect of absorving aerosol son global radiation Budget. Geophysical Research Letters. 1995; 22(8): 929-931.

3. Keil A, Haywood JM. Solar radiative forcing by biomass burning aerosol particles during SAFARI 2000: A case study based on measured aerosol and cloud properties. Journal of Geophysical Research. 2003; 108(D13): 1-18.

4. Chul EC, Ramanathanb V, Decremera D. Observationally constrained estimates of carbonaceous aerosol radiative forcing. 
Proceedings of the National Academy of Sciences (PNAS). 2012; 109(29): 11624-11629.

5. Servicio Nacional de Meteorología e Hidrología del Perú. Atlas climático de precipitación y temperature del aire en la cuenca del río Mantaro. Lima: SENAMHI / MINAM; 2011. Disponible en: http://sania.comunidadandina.org/UpLoad /Contenido/9/31/4\%20(PRAA)\%20Atlas\%20 de\%20Temperatura\%20y\%20Precipitacion.pdf

6. Instituto Geofísico Del Perú. Atlas Climático de precipitación y temperature del aire en la cuenca del río Mantaro. Lima: IGP / CONAM; 2005. Disponible en: http://www.met.igp.gob.pe/ publicaciones/2000_2007/Atlas_Climatico.pdf

7. Trasmonte G, Silvā Y, Segurā B, Latínez K. Variabilidad de las temperaturas máximas y mínimas en el valle del río Mantaro. Memoria del subproyecto "Pronóstico estacional de lluvias y temperature del aire en la cuenca del río Mantaro para su aplicación en la agricultura" (2007-2010). IGP [Internet]. 2010 [citado el 15 de octubre de 2015]; [15 paginas]. Disponible en: http://www.met.igp.gob.pe/publicaciones /2010/ trasmonteetal INCAGRO 2010.pdf

8. Arroyo J. Tendencias y cambio del clima del valle del Mantaro mediante los análisis de índices de precipitación efectiva y temperatura eficiente. Apunt. Cienc. Soc. 2011 ; 1(1): 45-54.

9. Robinson PJ. Measurements of downward scattered solar radiation from isolated Cumulus clouds. Journal of Applied Meteorology. 1977; 16: 620625.

10. Tunc S. Enhancement of solar and ultraviolet Surface irradiance under partial cloudy conditions. Utrecht: University of Utrecht / Institute of Marine and Atmospheric Research; 1999. Scientific Report WR 99-01. Disponible en: http://a.knmi2.nl/ bibliotheek/knmipubWR/WR1999-01.pdf

11. Camayo BF, Pomachagua JE, Massipe JR, Quispe MO. Validación y aplicación del modelo BristowCampbell para estimar la radiación solar global de la región de Junín. XX Simposio Peruano de Energía Solar. Tacna: Asociación Peruana de Energía Solar y del Ambiente; 2013. Disponible en: http://www. perusolar.org/wp-content/uploads/2013/12/ Becquer-Camayo-Lapa_paper2.pdf

12. Servicio Nacional de Meteorología e Hidrología. Atlas de Energía Solar del Perú. Lima: Ministerio de Energía y Minas / SENAMHI; 2003. Disponible en: http://www.senamhi.gob.pe/pdf/atlas_solar. pdf 